\title{
Stability Analysis of Impulsive Stochastic Reaction-Diffusion Cellular Neural Network with Distributed Delay via Fixed Point Theory
}

\author{
Ruofeng Rao' ${ }^{1}$ and Shouming Zhong ${ }^{2}$ \\ ${ }^{1}$ Department of Mathematics, Chengdu Normal University, Chengdu 61130, China \\ ${ }^{2}$ College of Mathematics, University of Electronic Science and Technology of China, Chengdu 611731, China \\ Correspondence should be addressed to Ruofeng Rao; ruofengrao@163.com
}

Received 14 July 2017; Accepted 22 August 2017; Published 25 September 2017

Academic Editor: Chenguang Yang

Copyright (c) 2017 Ruofeng Rao and Shouming Zhong. This is an open access article distributed under the Creative Commons Attribution License, which permits unrestricted use, distribution, and reproduction in any medium, provided the original work is properly cited.

\begin{abstract}
This paper investigates the stochastically exponential stability of reaction-diffusion impulsive stochastic cellular neural networks $(\mathrm{CNN})$. The reaction-diffusion pulse stochastic system model characterizes the complexity of practical engineering and brings about mathematical difficulties, too. However, the difficulties have been overcome by constructing a new contraction mapping and an appropriate distance on a product space which is guaranteed to be a complete space. This is the first time to employ the fixed point theorem to derive the stability criterion of reaction-diffusion impulsive stochastic CNN with distributed time delays. Finally, an example is provided to illustrate the effectiveness of the proposed methods.
\end{abstract}

\section{Introduction}

In 1988, cellular neural networks (CNN) were originally introduced in $[1,2]$. Since then, dynamic neural networks have received extensive attention due to their classification, associative memory, and parallel computing tasks and the ability to solve complex optimization problems. It is generally known that almost all neural networks have similar applications ([3-12]), but the key to the success of these applications lies in the stability of the system. In fact, there are a number of literatures involved in the stability analysis of CNN $([5,7,12-14])$. In practical engineering, time delay and pulse are unavoidable. Since neural networks usually have spatial properties, due to the existence of parallel paths of various axonal sizes and lengths, it is necessary to introduce continuous distributed delays to simulate them over a given time horizon. Besides, many evolutionary processes, especially the biological neural network in biological systems and bursting rhythm models in pathology, frequencymodulated signal processing systems, are characterized by abrupt changes of states at certain time instants. In addition, electrons have diffusion behavior in inhomogeneous media.
Noise disturbance is unavoidable in real nervous systems, which is a major source of instability and poor performance in neural networks. A neural network can be stabilized or destabilized by certain stochastic inputs. The synaptic transmission in real neural networks can be viewed as a noisy process introduced by random fluctuations from the release of neurotransmitters and other probabilistic causes. Hence, the above influent factors should be also taken into consideration in stability analysis of neural networks. So, in this paper, we consider a class of impulsive stochastic reaction-diffusion cellular neural networks with distributed delay. Lyapunov function method is one of the common techniques to solve the stability of neural networks in recent decades. However, every method has its limit. Different methods lead to different criteria for stability criteria which may imply innovations. Fixed point theory and method is one of the alternative methods ([15-22]). Unlike the known literature, we try to employ Banach fixed point theory in this paper to derive the stability of impulsive stochastic reactiondiffusion cellular neural networks with distributed delay. In the next sections, we shall give some model descriptions and preliminaries and employ Banach fixed point theorem, 
Hölder inequality, Burkholder-Davis-Gundy inequality, and the continuous semigroup of Laplace operators to derive the stochastically exponential stability criterion of the complex system. Of course, to overcome the difficulty of the complex mathematical model, we need to formulate a new contraction mapping on a product space. Moreover, in order to guarantee the completeness of product space, we need to give a reasonable definition of distance. Finally, an example is provided to illustrate the effectiveness of the proposed result.

\section{Model Description and Preliminaries}

Consider the following reaction-diffusion impulsive stochastic cellular neural networks under Dirichlet boundary value:

$$
\begin{aligned}
& d u_{i}(t, x)=-q_{i} \operatorname{div} \nabla u_{i}(t, x) d t-\left[a_{i} u_{i}(t, x)\right. \\
& -\sum_{j=1}^{n} b_{i j} f_{j}\left(u_{j}(t, x)\right)-\sum_{j=1}^{n} c_{i j} f_{j}\left(u_{j}(t-\tau(t), x)\right) \\
& \left.-\sum_{j=1}^{n} h_{i j} \int_{t-\rho(t)}^{t} f_{j}\left(u_{j}(s, x)\right) d s\right] d t \\
& +\sigma_{i}\left(u_{i}(t, x)\right) d w_{i}(t) \\
& t \neq t_{k}, x \in \Upsilon, \quad i \in \mathcal{N} \\
& u\left(t_{k}^{+}, x\right)=u\left(t_{k}^{-}, x\right)+g\left(u\left(t_{k}, x\right)\right), \\
& x \in \Upsilon, k=1,2, \ldots \\
& u_{i}(t, x)=\zeta_{i}(t, x), \quad \forall(s, x) \in[-\tau, 0] \times \Upsilon \\
& u(t, x)=0, \quad \forall(t, x) \in[0,+\infty) \times \partial \Upsilon,
\end{aligned}
$$

where $\Upsilon \subset R^{m}$ is a bounded domain with the smooth boundary $\partial Y . u_{i}(t, x)$ is the state variable of the $i$ th neuron at time $t$ and in space variable $x$ for $i \in \mathcal{N}$ with $\mathcal{N} \triangleq$ $\{1,2, \ldots, n\} . f_{i}$ denotes the active function of neuron. $a_{i}$ is the rate with which the $i$ th neuron will reset its potential to the resting state in isolation when disconnected from the networks and the external inputs. $b_{i j}, c_{i j}$, and $h_{i j}$ are elements of feedback template. Let $\left\{w_{i}(t), t \geqslant 0\right\}$ be a real-valued Brownian motion defined on the complete probability space $\{\Omega, \mathscr{F}, \mathbb{P}\}$ which has natural filtration $\left\{\mathscr{F}_{t}\right\}_{t \geqslant 0}$. Denote by $\mathscr{L}^{2}(\Upsilon)$ the space of all real-valued square integrable functions with the inner product $\langle\xi, \eta\rangle=\int_{\Upsilon} \xi(x) \eta(x) d x$, for $\xi, \eta \in$ $\mathscr{L}^{2}(\Upsilon)$ which derives the norm $\|\xi\|=\left(\int_{\Upsilon} \xi^{2}(x) d x\right)^{1 / 2}$ for $\xi \in \mathscr{L}^{2}(\Upsilon) . \sigma_{i}(\cdot)$ is a Borel measurable function. Denote by $\Delta=\sum_{j=1}^{m}\left(\partial^{2} / \partial x_{j}^{2}\right)$ the Laplace operator, with domain $\mathscr{D}(\Delta)=$ $W_{0}^{1,2}(\Upsilon) \cap W_{0}^{2,2}(\Upsilon)$, which generates a strongly continuous semigroup $e^{-q_{i} t \Delta}$, where $W_{0}^{1,2}(\Upsilon)$ and $W_{0}^{2,2}(\Upsilon)$ are the Sobolev spaces with compactly supported sets. $\operatorname{div} \nabla u_{i}(t, x)$ denotes the divergence of $\nabla u_{i}(t, x)$ (see, e.g., $\left.[25,26]\right) . q_{i}$ is the diffusion coefficient, and time delays $\tau(t), \rho(t) \in[0, \tau]$. Besides, initial value $\zeta_{i}(t, x)$ is continuous for $(s, x) \in[-\tau, 0] \times$ $\Upsilon$. The fixed impulsive moments $t_{k}(k=1,2, \ldots)$ satisfy $0<t_{1}<t_{2}<\cdots$ with $\lim _{k \rightarrow+\infty} t_{k}=+\infty \cdot u\left(t_{k}^{+}, x\right)$ and $u\left(t_{k}^{-}, x\right)$ stand for the right-hand and left-hand limit of $u(t, x)$ at time $t_{k}$, respectively. Further, suppose that $u\left(t_{k}^{-}, x\right)=$ $\lim _{t \rightarrow t_{k}^{-}} u(t, x)=u\left(t_{k}, x\right), k=1,2, \ldots$

In this paper, we assume that

(H1) $\left\|e^{-q_{i} t \Delta}\right\| \leqslant M e^{-\gamma t}$, where $M>0$ and $\gamma>0$ are constants;

(H2) $f_{i}, g_{i}$, and $\sigma_{i}$ are Lipschitz continuous with Lipschitz constants $L_{i}>0, G_{i}$, and $T_{i}>0$ for $i \in \mathcal{N}$, respectively. In addition, $f_{i}(0)=g_{i}(0)=0=\sigma_{i}(0), \forall i \in \mathcal{N}$.

Definition 1. For any $T>0$ and $x \in \Upsilon$, a stochastic process $u=\left\{\left(u_{1}(t, x), u_{2}(t, x), \ldots, u_{n}(t, x)\right)\right\}_{[0, T]}$ is called a mild solution of impulsive system (1) if, for any $i \in \mathcal{N}$, $u_{i}(t, x) \in \mathscr{C}\left([0, T] ; \mathscr{L}^{2}(\Upsilon)\right)$ and, for any $t \in[0, T], u_{i}(t, x)$ is adapted to $\mathscr{F}_{t}$ with

$$
\mathbb{P}\left\{\omega: \int_{0}^{t} \int_{\Upsilon}\left|u_{i}(s)\right|^{2} d x d s<\infty\right\}=1,
$$

and the following stochastic integral equations hold for all $i \in$ $\mathcal{N}$, a.s. for any $t \in[0, T]$ and $x \in \Upsilon$,

$$
\begin{aligned}
& u_{i}(t, x)=e^{-q_{i} t \Delta} \zeta(0, x)-\int_{0}^{t} e^{-q_{i}(t-\theta) \Delta}\left[a_{i} u_{i}(\theta, x)\right. \\
& \quad-\sum_{j=1}^{n} b_{i j} f_{j}\left(u_{j}(\theta, x)\right)-\sum_{j=1}^{n} c_{i j} f_{j}\left(u_{j}(\theta-\tau(\theta), x)\right) \\
& \left.\quad-\sum_{j=1}^{n} h_{i j} \int_{\theta-\rho(\theta)}^{\theta} f_{j}\left(u_{j}(s, x)\right) d s\right] d \theta \\
& +\int_{0}^{t} e^{-q_{i}(t-\theta) \Delta} \sigma_{i}\left(u_{i}(\theta, x)\right) d w_{i}(\theta) \\
& +e^{-q_{i} t \Delta} \sum_{0<t_{k}<t} e^{q_{i} t_{k} \Delta} g_{i}\left(u_{i}\left(t_{k}, x\right)\right), \quad t \geqslant 0 \\
& u_{i}(t, x)=\zeta_{i}(t, x), \quad \forall(s, x) \in[-\tau, 0] \times \Upsilon, \\
& u(t, x)=0, \quad \forall(t, x) \in[0,+\infty) \times \partial \Upsilon .
\end{aligned}
$$

Remark 2. In Definition 1, the mild solution of impulsive system (1) is well defined due to [24, Lemma 3.1].

Lemma 3 (Hölder inequality). Assume that $1 / p+1 / q=1$ with $p>1$, and $\varphi(x) \in \mathscr{L}^{p}(\Upsilon), \phi \in \mathscr{L}^{q}(\Upsilon)$; then,

$$
\begin{aligned}
& \int_{\Upsilon} \varphi(x) \phi(x) d x \\
& \quad \leqslant\left(\int_{\Upsilon}|\varphi(x)|^{p} d x\right)^{1 / p}\left(\int_{\Upsilon}|\phi(x)|^{q} d x\right)^{1 / q} .
\end{aligned}
$$

Lemma 4 (Banach contraction mapping principle). Let $\Theta$ be a contraction operator on a complete metric space $\Gamma$; then there exists a unique point $u \in \Gamma$ for which $\Theta(u)=u$. 


\section{Main Result: Stochastically Exponential Stability}

Theorem 5. Assume that (H1) and (H2) hold. Then, CNN (1) is stochastically exponentially mean square stable if the following condition holds:

$$
0<\kappa<1
$$

where $\mu=\inf _{k=1,2, \ldots}\left(t_{k+1}-t_{k}\right)>0$ and

$$
\begin{aligned}
\kappa \triangleq & 6 M^{2}\left[\frac{1}{\gamma^{2}}\left(\max _{i \in \mathcal{N}} a_{i}^{2}\right)\right. \\
& +n \frac{1}{\gamma^{2}} \max _{i \in \mathcal{N}}\left(\sum_{j=1}^{n}\left(\left|b_{i j}\right|^{2}+\left|c_{i j}\right|^{2}\right) L_{j}^{2}\right)+\frac{n \tau^{2}}{\gamma^{2}} \\
& \left.+2 M^{2}\left(1+\frac{1}{\gamma^{2} \mu^{2}}\right)\left(\max _{i \in \mathcal{N}} G_{i}^{2}\right)+\frac{2}{\gamma}\left(\max _{i \in \mathcal{N}} T_{i}^{2}\right)\right] .
\end{aligned}
$$

Proof. Firstly, we need to formulate a contraction mapping on a product space.

Let $\Gamma_{i}$ be the Banach space of all $\mathscr{F}_{t}$-adapted mean square continuous processes consisting of functions $u_{i}(t, x)$ at $t \geqslant 0$ with $t \neq t_{k}$ such that $\mathbb{E}\left(e^{\alpha t}\left\|u_{i}(t, x)\right\|^{2}\right) \rightarrow 0$ as $t \rightarrow+\infty$, where $\alpha \in(0, \gamma)$ is a positive scalar. Now, we construct an operator $\Theta \triangleq\left(\Theta_{1}, \Theta_{2}, \ldots, \Theta_{i}, \ldots, \Theta_{n}\right)$ with $\Theta_{i}: \Gamma_{i} \rightarrow \Gamma_{i}$ as follows:

$$
\begin{aligned}
& \Theta_{i}\left(u_{i}\right)(t, x)=e^{-q_{i} t \Delta} \zeta(0, x)-\int_{0}^{t} e^{-q_{i}(t-\theta) \Delta}\left[a_{i} u_{i}(\theta, x)\right. \\
& -\sum_{j=1}^{n} b_{i j} f_{j}\left(u_{j}(\theta, x)\right)-\sum_{j=1}^{n} c_{i j} f_{j}\left(u_{j}(\theta-\tau(\theta), x)\right) \\
& \left.\quad-\sum_{j=1}^{n} h_{i j} \int_{\theta-\rho(\theta)}^{\theta} f_{j}\left(u_{j}(s, x)\right) d s\right] d \theta \\
& +\int_{0}^{t} e^{-q_{i}(t-\theta) \Delta} \sigma_{i}\left(u_{i}(\theta, x)\right) d w_{i}(\theta) \\
& +e^{-q_{i} t \Delta} \sum_{0<t_{k}<t} e^{q_{i} t_{k} \Delta} g_{i}\left(u_{i}\left(t_{k}, x\right)\right), \quad t \geqslant 0, \\
& \Theta_{i}\left(u_{i}\right)(t, x)=\zeta_{i}(t, x), \quad(s, x) \in[-\tau, 0] \times \Upsilon \\
& \Theta_{i}\left(u_{i}\right)(t, x)=0, \quad \forall(t, x) \in[0,+\infty) \times \partial \Upsilon .
\end{aligned}
$$

Equipped with the following distance:

$$
\begin{array}{r}
\operatorname{dist}(u, v)=\left(\mathbb{E} \max _{i \in \mathcal{N}} \sup _{t \geqslant-\tau}\left\|u_{i}(t, x)-v_{i}(t, x)\right\|^{2}\right)^{1 / 2}, \\
\forall u, v \in \Gamma_{1} \times \Gamma_{2} \times \cdots \times \Gamma_{n},
\end{array}
$$

$\Gamma_{1} \times \Gamma_{2} \times \cdots \times \Gamma_{n}$ becomes a complete metric space, where $u=u(t, x)=\left(u_{1}(t, x), u_{2}(t, x), \ldots, u_{n}(t, x)\right)^{T}, v=v(t, x)=$ $\left(v_{1}(t, x), v_{2}(t, x), \ldots, v_{n}(t, x)\right)^{T}$.
Next, we are to apply contractive mapping theory to complete the proof via three steps.

Step 1. From (7), for $t \in[0,+\infty) \backslash\left\{t_{k}\right\}_{k=1}^{\infty}$, we claim that $\Theta_{i}\left(u_{i}\right)(t)$ is mean square continuous. Indeed, let $\delta$ be a small enough scalar:

$$
\begin{aligned}
& \mathbb{E}\left\|\Theta_{i}\left(u_{i}\right)(t+\delta, x)-\Theta_{i}\left(u_{i}\right)(t, x)\right\|^{2} \\
& \leqslant 4 \mathbb{E}\left\|e^{-q_{i}(t+\delta) \Delta} \zeta(0, x)-e^{-q_{i} t \Delta} \zeta(0, x)\right\|^{2} \\
& +4 \mathbb{E} \| \int_{0}^{t+\delta} e^{-q_{i}(t+\delta-\theta) \Delta}\left[a_{i} u_{i}(\theta, x)\right. \\
& -\sum_{j=1}^{n} b_{i j} f_{j}\left(u_{j}(\theta, x)\right)-\sum_{j=1}^{n} c_{i j} f_{j}\left(u_{j}(\theta-\tau(\theta), x)\right) \\
& \left.-\sum_{j=1}^{n} h_{i j} \int_{\theta-\rho(\theta)}^{\theta} f_{j}\left(u_{j}(s, x)\right) d s\right] d \theta \\
& -\int_{0}^{t} e^{-q_{i}(t-\theta) \Delta}\left[a_{i} u_{i}(\theta, x)-\sum_{j=1}^{n} b_{i j} f_{j}\left(u_{j}(\theta, x)\right)\right. \\
& -\sum_{j=1}^{n} c_{i j} f_{j}\left(u_{j}(\theta-\tau(\theta), x)\right) \\
& \left.-\sum_{j=1}^{n} h_{i j} \int_{\theta-\rho(\theta)}^{\theta} f_{j}\left(u_{j}(s, x)\right) d s\right] d \theta \|^{2} \\
& +4 \mathbb{E} \| \int_{0}^{t+\delta} e^{-q_{i}(t+\delta-\theta) \Delta} \sigma_{i}\left(u_{i}(\theta, x)\right) d w_{i}(\theta) \\
& -\int_{0}^{t} e^{-q_{i}(t-\theta) \Delta} \sigma_{i}\left(u_{i}(\theta, x)\right) d w_{i}(\theta) \|^{2} \\
& +4 \mathbb{E} \| e^{-q_{i}(t+\delta) \Delta} \sum_{0<t_{k}<t+\delta} e^{q_{i} t_{k} \Delta} g_{i}\left(u_{i}\left(t_{k}, x\right)\right) \\
& -e^{-q_{i} t \Delta} \sum_{0<t_{k}<t} e^{q_{i} t_{k} \Delta} g_{i}\left(u_{i}\left(t_{k}, x\right)\right) \|^{2} .
\end{aligned}
$$

Firstly, we estimate

$$
\begin{aligned}
& \mathbb{E}\left\|e^{-q_{i}(t+\delta) \Delta} \zeta(0, x)-e^{-q_{i} t \Delta} \zeta(0, x)\right\|^{2} \\
& \quad \leqslant \mathbb{E}\left\|\left(e^{-q_{i} \delta \Delta}-1\right) e^{-q_{i} t \Delta} \zeta(0, x)\right\|^{2} \longrightarrow 0,
\end{aligned}
$$

Next, we evaluate

$$
\begin{aligned}
& \mathbb{E} \| \int_{0}^{t+\delta} e^{-q_{i}(t+\delta-\theta) \Delta}\left[a_{i} u_{i}(\theta, x)-\sum_{j=1}^{n} b_{i j} f_{j}\left(u_{j}(\theta, x)\right)\right. \\
& \quad-\sum_{j=1}^{n} c_{i j} f_{j}\left(u_{j}(\theta-\tau(\theta), x)\right)
\end{aligned}
$$




$$
\begin{aligned}
& \left.-\sum_{j=1}^{n} h_{i j} \int_{\theta-\rho(\theta)}^{\theta} f_{j}\left(u_{j}(s, x)\right) d s\right] d \theta \\
& -\int_{0}^{t} e^{-q_{i}(t-\theta) \Delta}\left[a_{i} u_{i}(\theta, x)-\sum_{j=1}^{n} b_{i j} f_{j}\left(u_{j}(\theta, x)\right)\right. \\
& -\sum_{j=1}^{n} c_{i j} f_{j}\left(u_{j}(\theta-\tau(\theta), x)\right) \\
& \left.-\sum_{j=1}^{n} h_{i j} \int_{\theta-\rho(\theta)}^{\theta} f_{j}\left(u_{j}(s, x)\right) d s\right] d \theta \|^{2} \\
& \leqslant 2 \mathbb{E} \| \int_{t}^{t+\delta} e^{-q_{i}(t+\delta-\theta) \Delta}\left[a_{i} u_{i}(\theta, x)\right. \\
& -\sum_{j=1}^{n} b_{i j} f_{j}\left(u_{j}(\theta, x)\right)-\sum_{j=1}^{n} c_{i j} f_{j}\left(u_{j}(\theta-\tau(\theta), x)\right) \\
& \left.-\sum_{j=1}^{n} h_{i j} \int_{\theta-\rho(\theta)}^{\theta} f_{j}\left(u_{j}(s, x)\right) d s\right] d \theta \|^{2} \\
& +2 \mathbb{E} \| \int_{0}^{t} e^{-q_{i}(t-\theta) \Delta}\left[a_{i} u_{i}(\theta, x)-\sum_{j=1}^{n} b_{i j} f_{j}\left(u_{j}(\theta, x)\right)\right. \\
& -\sum_{j=1}^{n} c_{i j} f_{j}\left(u_{j}(\theta-\tau(\theta), x)\right) \\
& \left.-\sum_{j=1}^{n} h_{i j} \int_{\theta-\rho(\theta)}^{\theta} f_{j}\left(u_{j}(s, x)\right) d s\right]\left(e^{-q_{i} \delta \Delta}-1\right) d \theta \|^{2} \\
& \longrightarrow 0, \quad \text { if } \delta \longrightarrow 0 \text {. }
\end{aligned}
$$

Via Burkholder-Davis-Gundy inequality, we can conclude that if $\delta \rightarrow 0$,

$$
\begin{aligned}
& \mathbb{E} \| \int_{0}^{t+\delta} e^{-q_{i}(t+\delta-\theta) \Delta} \sigma_{i}\left(u_{i}(\theta, x)\right) d w_{i}(\theta) \\
& -\int_{0}^{t} e^{-q_{i}(t-\theta) \Delta} \sigma_{i}\left(u_{i}(\theta, x)\right) d w_{i}(\theta) \|^{2} \\
& \quad \leqslant 8 \mathbb{E} \int_{0}^{t} M^{2} e^{-2 \gamma(t-\theta)}\left\|\sigma_{i}\left(u_{i}(\theta, x)\right)\left(e^{-q_{i} \delta \Delta}-1\right)\right\|^{2} d \theta \\
& +8 \mathbb{E} \int_{t}^{t+\delta} M^{2} e^{-2 \gamma(t+\delta-\theta)}\left\|\sigma_{i}\left(u_{i}(\theta, x)\right)\right\|^{2} d \theta \longrightarrow 0 .
\end{aligned}
$$

Due to $t \neq t_{k}$, it is obvious that

$$
\begin{gathered}
\mathbb{E} \| e^{-q_{i}(t+\delta) \Delta} \sum_{0<t_{k}<t+\delta} e^{q_{i} t_{k} \Delta} g_{i}\left(u_{i}\left(t_{k}, x\right)\right) \\
-e^{-q_{i} t \Delta} \sum_{0<t_{k}<t} e^{q_{i} t_{k} \Delta} g_{i}\left(u_{i}\left(t_{k}, x\right)\right) \|^{2} \longrightarrow 0,
\end{gathered}
$$

if $\delta \longrightarrow 0$.

So, we have proved from $(10)-(14)$ that $\Theta_{i}\left(u_{i}\right)(t)$ is mean square continuous at $t \geqslant 0$ with $t \neq t_{k}$.

Next, we claim that

$$
\begin{aligned}
& \lim _{\delta \rightarrow 0^{+}} \Theta_{i}\left(u_{i}\right)\left(t_{k}+\delta\right)=\Theta_{i}\left(u_{i}\right)\left(t_{k}\right)+g\left(u_{i}\left(t_{k}\right)\right), \\
& \lim _{\delta \rightarrow 0^{-}} \Theta_{i}\left(u_{i}\right)\left(t_{k}+\delta\right)=\Theta_{i}\left(u_{i}\right)\left(t_{k}\right) .
\end{aligned}
$$

Indeed, obviously, (11)-(13) hold for all $t=t_{k}$, too. In addition, let $\delta>0$ be small enough:

$$
\begin{gathered}
e^{-q_{i}\left(t_{k}+\delta\right) \Delta} \sum_{0<t_{j}<t_{k}+\delta} e^{q_{i} t_{j} \Delta} g_{i}\left(u_{i}\left(t_{j}, x\right)\right) \\
-e^{-q_{i} t_{k} \Delta} \sum_{0<t_{j}<t_{k}} e^{q_{i} t_{j} \Delta} g_{i}\left(u_{i}\left(t_{j}, x\right)\right) \\
=g_{i}\left(u\left(t_{k}, x\right)\right), \quad \delta \longrightarrow 0^{+} .
\end{gathered}
$$

On the other hand, let $\delta<0$ be small enough:

$$
\begin{gathered}
e^{-q_{i}\left(t_{k}+\delta\right) \Delta} \sum_{0<t_{j}<t_{k}+\delta} e^{q_{i} t_{j} \Delta} g_{i}\left(u_{i}\left(t_{j}, x\right)\right) \\
-e^{-q_{i} t_{k} \Delta} \sum_{0<t_{j}<t_{k}} e^{q_{i} t_{j} \Delta} g_{i}\left(u_{i}\left(t_{j}, x\right)\right)=0,
\end{gathered}
$$

$$
\delta \longrightarrow 0^{-}
$$

This together with (16) implies that (15) holds.

Step 2. We claim that

$$
\mathbb{E}\left(e^{\alpha t}\left\|\Theta_{i}\left(u_{i}(t, x)\right)\right\|^{2}\right) \longrightarrow 0, \quad \text { if } t \longrightarrow+\infty
$$

Indeed, we have the following inequality similar to (10):

$$
\begin{aligned}
& \mathbb{E}\left(e^{\alpha t}\left\|\Theta_{i}\left(u_{i}\right)(t, x)\right\|^{2}\right) \leqslant 4 \mathbb{E}\left(e^{\alpha t}\left\|e^{-q_{i} t \Delta} \zeta(0, x)\right\|^{2}\right) \\
& +4 \mathbb{E}\left\{e^{\alpha t} \| \int_{0}^{t} e^{-q_{i}(t-\theta) \Delta}\left[a_{i} u_{i}(\theta, x)\right.\right.
\end{aligned}
$$




$$
\begin{aligned}
& -\sum_{j=1}^{n} b_{i j} f_{j}\left(u_{j}(\theta, x)\right)-\sum_{j=1}^{n} c_{i j} f_{j}\left(u_{j}(\theta-\tau(\theta), x)\right) \\
& \left.\left.-\sum_{j=1}^{n} h_{i j} \int_{\theta-\rho(\theta)}^{\theta} f_{j}\left(u_{j}(s, x)\right) d s\right] d \theta\|\|^{2}\right\} \\
& +4 \mathbb{E}\left(e^{\alpha t}\left\|\int_{0}^{t} e^{-q_{i}(t-\theta) \Delta} \sigma_{i}\left(u_{i}(\theta, x)\right) d w_{i}(\theta)\right\|^{2}\right) \\
& +4 \mathbb{E}\left(e^{\alpha t}\left\|e^{-q_{i} t \Delta} \sum_{0<t_{k}<t} e^{q_{i} t_{k} \Delta} g_{i}\left(u_{i}\left(t_{k}, x\right)\right)\right\|^{2}\right)
\end{aligned}
$$

$t \geqslant 0$

(19)

Condition (H1) yields

$$
\begin{aligned}
& \mathbb{E}\left(e^{\alpha t}\left\|e^{-q_{i} t \Delta} \zeta(0, x)\right\|^{2}\right) \leqslant \mathbb{E}\left(M^{2} e^{-(2 \gamma-\alpha) t}\|\zeta(0, x)\|^{2}\right) \\
& \longrightarrow 0, \quad \text { if } t \longrightarrow+\infty
\end{aligned}
$$

For any given $\varepsilon>0$, the assumption $\mathbb{E}\left(e^{\alpha t}\left\|u_{i}(t, x)\right\|^{2}\right) \rightarrow 0$ tells us that there exists $t_{*}>0$ such that

$$
\mathbb{E}\left(e^{\alpha t}\left\|u_{i}(t, x)\right\|^{2}\right)<\varepsilon, \quad \forall t \geqslant t_{*} .
$$

Moreover, Hölder inequality gives

$$
\begin{aligned}
\mathbb{E}\left(e^{\alpha t}\left\|\int_{0}^{t} e^{-q_{i}(t-\theta) \Delta} a_{i} u_{i}(\theta, x) d \theta\right\|^{2}\right) \leqslant \frac{M^{2} a_{i}^{2}}{\gamma} \\
\cdot \mathbb{E}\left(e^{\alpha t} \int_{0}^{t} e^{-\gamma(t-\theta)}\left\|u_{i}(\theta, x)\right\|^{2} d \theta\right) \leqslant \frac{M^{2} a_{i}^{2}}{\gamma} \\
\cdot \mathbb{E}\left(e^{-(\gamma-\alpha) t} t^{*} e^{\gamma t^{*}} \max _{\theta \in\left[0, t_{*}\right]}\left(\left\|u_{i}(\theta, x)\right\|^{2}\right)+\varepsilon \frac{1}{\gamma-\alpha}\right),
\end{aligned}
$$

which together with the arbitrariness of $\varepsilon$ derives

$$
\begin{aligned}
\mathbb{E}\left(e^{\alpha t}\left\|\int_{0}^{t} e^{-q_{i}(t-\theta) \Delta} a_{i} u_{i}(\theta, x) d \theta\right\|^{2}\right) & \longrightarrow \\
& \text { if } t \longrightarrow+\infty .
\end{aligned}
$$

Besides,

$$
\begin{aligned}
& \mathbb{E}\left(e^{\alpha t}\left\|\int_{0}^{t} e^{-q_{i}(t-\theta) \Delta} \sum_{j=1}^{n} b_{i j} f_{j}\left(u_{j}(\theta, x)\right) d \theta\right\|^{2}\right) \\
& \leqslant \mathbb{E}\left(M \sum_{j=1}^{n}\left|b_{i j}\right| L_{j} e^{-(\gamma-\alpha) t} \int_{0}^{t} e^{\gamma \theta}\left\|u_{j}(\theta, x)\right\| d \theta\right)^{2} .
\end{aligned}
$$

Using similar methods of (21) and (22), we can deduce from (24) that

$$
\mathbb{E}\left(e^{\alpha t}\left\|\int_{0}^{t} e^{-q_{i}(t-\theta) \Delta} \sum_{j=1}^{n} b_{i j} f_{j}\left(u_{j}(\theta, x)\right) d \theta\right\|^{2}\right) \longrightarrow 0,
$$$$
\text { if } t \rightarrow+\infty \text {. }
$$

Similar to that of (24) and (22), we can also obtain

$$
\begin{gathered}
\mathbb{E}\left(e^{\alpha t}\left\|\int_{0}^{t} e^{-q_{i}(t-\theta) \Delta} \sum_{j=1}^{n} c_{i j} f_{j}\left(u_{j}(\theta-\tau(\theta), x)\right) d \theta\right\|^{2}\right) \\
\leqslant \mathbb{E}\left[M \sum _ { j = 1 } ^ { n } | c _ { i j } | L _ { j } \frac { 1 } { \gamma } \left(e^{-(\gamma-\alpha) t} e^{\gamma \tau}\left(t_{*}+\tau\right)\right.\right. \\
\left.\left.. \max _{s \in\left[-\tau, t^{*}+\tau\right]}\left(e^{\gamma s}\left\|u_{j}(s, x)\right\|^{2}\right)+\varepsilon e^{\gamma \tau} \frac{1}{\gamma-\alpha}\right)\right] .
\end{gathered}
$$

Now, similar to that of (22), we know from (26) that

$$
\begin{aligned}
& \mathbb{E}\left(e^{\alpha t}\left\|\int_{0}^{t} e^{-q_{i}(t-\theta) \Delta} \sum_{j=1}^{n} c_{i j} f_{j}\left(u_{j}(\theta-\tau(\theta), x)\right) d \theta\right\|^{2}\right) \\
& \longrightarrow 0, \quad \text { if } t \longrightarrow+\infty .
\end{aligned}
$$

Similarly, Hölder inequality yields

$$
\begin{aligned}
& \mathbb{E}\left(e^{\alpha t}\left\|\int_{0}^{t} e^{-q_{i}(t-\theta) \Delta} \sum_{j=1}^{n} h_{i j} \int_{\theta-\rho(\theta)}^{\theta} f_{j}\left(u_{j}(s, x)\right) d s d \theta\right\|^{2}\right) \\
& \leqslant M^{2} \mathbb{E}\left[\sum_{j=1}^{n}\left|h_{i j}\right| L_{j} \frac{\tau}{\gamma}\right. \\
& \cdot\left(e^{-(\gamma-\alpha) t} \tau \max _{\theta \in\left[-\tau, t_{*}+\tau\right]}\left\|u_{j}(s, x)\right\|^{2} \frac{1}{\gamma} e^{\gamma\left(t_{*}+\tau\right)}+\varepsilon \tau e^{\alpha \tau}\right. \\
& \left.\left.\cdot \frac{1}{\gamma-\alpha}\right)\right] .
\end{aligned}
$$

Similar to (22), we can conclude from (28) that

$$
\mathbb{E}\left(e^{\alpha t}\left\|\int_{0}^{t} e^{-q_{i}(t-\theta) \Delta} \sum_{j=1}^{n} h_{i j} \int_{\theta-\rho(\theta)}^{\theta} f_{j}\left(u_{j}(s, x)\right) d s d \theta\right\|^{2}\right)
$$

$\longrightarrow 0$, if $t \longrightarrow+\infty$.

Hence,

$$
\begin{aligned}
\mathbb{E} & \left\{e^{\alpha t} \| \int_{0}^{t} e^{-q_{i}(t-\theta) \Delta}\left[a_{i} u_{i}(\theta, x)-\sum_{j=1}^{n} b_{i j} f_{j}\left(u_{j}(\theta, x)\right)\right.\right. \\
& -\sum_{j=1}^{n} c_{i j} f_{j}\left(u_{j}(\theta-\tau(\theta), x)\right) \\
& \left.\left.-\sum_{j=1}^{n} h_{i j} \int_{\theta-\rho(\theta)}^{\theta} f_{j}\left(u_{j}(s, x)\right) d s\right] d \theta \|^{2}\right\}
\end{aligned}
$$




$$
\begin{aligned}
& \leqslant 4 \mathbb{E}\left\|e^{\alpha t} \int_{0}^{t} e^{-q_{i}(t-\theta) \Delta} a_{i} u_{i}(\theta, x) d \theta\right\|^{2} \\
& +4 \mathbb{E}\left\|e^{\alpha t} \int_{0}^{t} e^{-q_{i}(t-\theta) \Delta} \sum_{j=1}^{n} b_{i j} f_{j}\left(u_{j}(\theta, x)\right) d \theta\right\|^{2} \\
& +4 \mathbb{E} \| e^{\alpha t} \int_{0}^{t} e^{-q_{i}(t-\theta) \Delta} \sum_{j=1}^{n} c_{i j} f_{j}\left(u_{j}(\theta\right. \\
& -\tau(\theta), x)) d \theta\left\|^{2}+4 \mathbb{E}\right\| e^{\alpha t} \int_{0}^{t} e^{-q_{i}(t-\theta) \Delta} \sum_{j=1}^{n} h_{i j} \\
& \cdot \int_{\theta-\rho(\theta)}^{\theta} f_{j}\left(u_{j}(s, x)\right) d s d \theta \|^{2} \longrightarrow 0,
\end{aligned}
$$$$
\text { if } t \longrightarrow+\infty \text {. }
$$

Burkholder-Davis-Gundy inequality and Hölder inequality derive

$$
\begin{aligned}
& \mathbb{E}\left(e^{\alpha t}\left\|\int_{0}^{t} e^{-q_{i}(t-\theta) \Delta} \sigma_{i}\left(u_{i}(\theta, x)\right) d w_{i}(\theta)\right\|^{2}\right) \\
& \leqslant 8 \mathbb{E}\left(M^{2} T_{i}^{2} e^{-(2 \gamma-\alpha) t} t_{*} \max _{\theta \in\left[0, t^{*}\right]} e^{2 \gamma \theta}\left\|u_{i}(\theta, x)\right\|^{2}\right) \\
& +8 \varepsilon \mathbb{E}\left(M^{2} T_{i}^{2} \sqrt{\frac{1}{4 \gamma(\gamma-\alpha)}}\right)
\end{aligned}
$$

which together with the arbitrariness of $\varepsilon$ implies

$$
\begin{aligned}
& \mathbb{E}\left(e^{\alpha t}\left\|\int_{0}^{t} e^{-q_{i}(t-\theta) \Delta} \sigma_{i}\left(u_{i}(\theta, x)\right) d w_{i}(\theta)\right\|^{2}\right) \longrightarrow 0 \\
& \text { if } t \longrightarrow+\infty
\end{aligned}
$$

Next, we may assume that $t_{l-1}<t_{*} \leqslant t_{l}$ and $t_{j-1}<t \leqslant t_{j}$. In addition, one can deduce from (H1)

$$
\begin{aligned}
& \mathbb{E}\left\{e^{\alpha t}\left\|e^{-q_{i} t \Delta} \sum_{0<t_{k} \leqslant t_{*}} e^{q_{i} t_{k} \Delta} g_{i}\left(u_{i}\left(t_{k}, x\right)\right)\right\|^{2}\right\} \\
& \leqslant \mathbb{E}\left[M e^{(\alpha / 2) t} e^{-\gamma t}\left(\sum_{0<t_{k} \leqslant t_{l}} M e^{\gamma t_{k}} G_{i}\left\|u_{i}\left(t_{k}, x\right)\right\|\right)\right]^{2}
\end{aligned}
$$

Besides, we can estimate by means of definite integral

$$
\begin{aligned}
& \mathbb{E}\left\{e^{\alpha t}\left\|e^{-q_{i} t \Delta} \sum_{t_{*}<t_{k}<t} e^{q_{i} t_{k} \Delta} g_{i}\left(u_{i}\left(t_{k}, x\right)\right)\right\|^{2}\right\} \\
& \leqslant \varepsilon G_{i}^{2} M^{4} \mathbb{E}\left(e^{-(1 / 2)(2 \gamma-\alpha) t} \sum_{t_{l} \leqslant t_{k} \leqslant t_{j-1}} e^{(1 / 2)(2 \gamma-\alpha) t_{k}}\right)^{2} \\
& \leqslant \varepsilon \mathbb{E}\left(M^{2} G_{i}\left(1+\frac{2}{\mu(2 \gamma-\alpha)}\right)\right)^{2} .
\end{aligned}
$$

Moreover, the arbitrariness of $\varepsilon$ implies

$$
\begin{aligned}
\mathbb{E}\left\{e^{\alpha t}\left\|e^{-q_{i} t \Delta} \sum_{t_{*}<t_{k}<t} e^{q_{i} t_{k} \Delta} g_{i}\left(u_{i}\left(t_{k}, x\right)\right)\right\|^{2}\right\} & \longrightarrow \\
\text { if } t \longrightarrow+\infty & 0,
\end{aligned}
$$

Hence, if $t \rightarrow+\infty$,

$$
\begin{aligned}
& \mathbb{E}\left\{e^{\alpha t}\left\|e^{-q_{i} t \Delta} \sum_{0<t_{k}<t} e^{q_{i} t_{k} \Delta} g_{i}\left(u_{i}\left(t_{k}, x\right)\right)\right\|^{2}\right\} \\
& \leqslant 2 \mathbb{E}\left(e^{\alpha t}\left\|e^{-q_{i} t \Delta} \sum_{0<t_{k} \leqslant t_{*}} e^{q_{i} t_{k} \Delta} g_{i}\left(u_{i}\left(t_{k}, x\right)\right)\right\|^{2}\right) \\
& +2 \mathbb{E}\left(e^{\alpha t}\left\|e^{-q_{i} t \Delta} \sum_{t_{*}<t_{k}<t} e^{q_{i} t_{k} \Delta} g_{i}\left(u_{i}\left(t_{k}, x\right)\right)\right\|^{2}\right) \\
& \longrightarrow 0 .
\end{aligned}
$$

Combining (19), (20), (23), (30), (32), and (36) results in (18).

Step 3. Finally, we claim that $\Theta$ is a contractive mapping on $\Gamma_{1} \times \Gamma_{2} \times \cdots \times \Gamma_{n}$.

Indeed, from the above two steps, we know $\Theta_{i}\left(\Gamma_{i}\right) \subset \Gamma_{i}$, and then $\Theta\left(\Gamma_{1} \times \Gamma_{2} \times \cdots \times \Gamma_{n}\right) \subset \Gamma_{1} \times \Gamma_{2} \times \cdots \times \Gamma_{n}$.

On the other hand, for any $i \in \mathcal{N}$ and $u, v \in \Gamma_{1} \times \Gamma_{2} \times \cdots \times \Gamma_{n}$,

$$
\begin{aligned}
& \mathbb{E} \max _{i \in \mathcal{N}} \sup _{t \geqslant-\tau}\left\|\Theta_{i}\left(u_{i}\right)(t, x)-\Theta_{i}\left(v_{i}\right)(t, x)\right\|^{2} \\
& \leqslant 6 \mathbb{E} \max _{i \in \mathcal{N}} \sup _{t \geqslant-\tau} \| \int_{0}^{t} e^{-q_{i}(t-\theta) \Delta} a_{i}\left(u_{i}(\theta, x)\right. \\
& \left.-v_{i}(\theta, x)\right) d \theta\left\|^{2}+6 \mathbb{E} \max _{i \in \mathcal{N}} \sup _{t \geqslant-\tau}\right\| \int_{0}^{t} e^{-q_{i}(t-\theta) \Delta} \\
& \cdot \sum_{j=1}^{n} b_{i j}\left(f_{j}\left(u_{j}(\theta, x)\right)-f_{j}\left(v_{j}(\theta, x)\right)\right) d \theta \|^{2}
\end{aligned}
$$




$$
\begin{aligned}
& +6 \mathbb{E} \max _{i \in \mathcal{N}} \sup _{t \geqslant-\tau} \| \int_{0}^{t} e^{-q_{i}(t-\theta) \Delta} \sum_{j=1}^{n} c_{i j} \\
& \cdot\left(f_{j}\left(u_{j}(\theta-\tau(\theta), x)\right)\right. \\
& \left.-f_{j}\left(v_{j}(\theta-\tau(\theta), x)\right)\right) d \theta \|^{2} \\
& +6 \mathbb{E} \max _{i \in \mathcal{N}} \sup _{t \geqslant-\tau} \| \int_{0}^{t} e^{-q_{i}(t-\theta) \Delta} \sum_{j=1}^{n} h_{i j} \\
& \cdot\left(\int_{\theta-\rho(\theta)}^{\theta}\left[f_{j}\left(u_{j}(s, x)\right)-f_{j}\left(v_{j}(s, x)\right)\right] d s\right) d \theta \|^{2} \\
& +6 \mathbb{E} \max _{i \in \mathcal{N}} \sup _{t \geqslant-\tau} \| \int_{0}^{t} e^{-q_{i}(t-\theta) \Delta}\left[\sigma_{i}\left(u_{i}(\theta, x)\right)\right. \\
& \left.-\sigma_{i}\left(v_{i}(\theta, x)\right)\right] d w_{i}(\theta)\|\|^{2} \\
& +6 \mathbb{E} \max _{i \in \mathscr{N}} \sup _{t \geqslant-\tau} \| e^{-q_{i} t \Delta} \sum_{0<t_{k}<t} e^{q_{i} t_{k} \Delta}\left[g_{i}\left(u_{i}\left(t_{k}, x\right)\right)\right. \\
& \left.-g_{i}\left(v_{i}\left(t_{k}, x\right)\right)\right] \| \cdot
\end{aligned}
$$

Besides, it follows by the Hölder inequality that

$$
\begin{aligned}
& \mathbb{E} \max _{i \in \mathcal{N}} \sup _{t \geqslant-\tau}\left\|\int_{0}^{t} e^{-q_{i}(t-\theta) \Delta} a_{i}\left(u_{i}(\theta, x)-v_{i}(\theta, x)\right) d \theta\right\|^{2} \leqslant M^{2} \\
& \cdot \frac{1}{\gamma^{2}}\left(\max _{i \in \mathcal{N}} a_{i}^{2}\right) \mathbb{E} \max _{i \in \mathcal{N}} \sup _{t \geqslant-\tau}\left\|u_{i}(\theta, x)-v_{i}(\theta, x)\right\|^{2}, \\
& \mathbb{E} \max _{i \in \mathcal{N}} \sup _{t \geqslant-\tau} \| \int_{0}^{t} e^{-q_{i}(t-\theta) \Delta} \sum_{j=1}^{n} b_{i j} \\
& \cdot\left(f_{j}\left(u_{j}(\theta, x)\right)-f_{j}\left(v_{j}(\theta, x)\right)\right) d \theta \|^{2} \leqslant n M^{2} \frac{1}{\gamma^{2}} \\
& \cdot \max _{i \in \mathcal{N}}\left(\sum_{j=1}^{n}\left|b_{i j}\right|^{2} L_{j}^{2}\right) \mathbb{E} \max _{i \in \mathcal{N}} \sup _{t \geqslant-\tau}\left\|u_{i}(t, x)-v_{i}(t, x)\right\|^{2}, \\
& \mathbb{E} \max _{i \in \mathcal{N}} \sup _{t \geqslant-\tau} \| \int_{0}^{t} e^{-q_{i}(t-\theta) \Delta} \sum_{j=1}^{n} c_{i j} \\
& \cdot\left(f_{j}\left(u_{j}(\theta-\tau(\theta), x)\right)-f_{j}\left(v_{j}(\theta-\tau(\theta), x)\right)\right) d \theta \|^{2} \\
& \quad \leqslant n M^{2} \frac{1}{\gamma^{2}} \max _{i \in \mathcal{N}}\left(\sum_{j=1}^{n}\left|c_{i j}\right|^{2} L_{j}^{2}\right) \mathbb{E} \max _{i \in \mathcal{N}} \sup _{t \geqslant-\tau} \| u_{i}(t, x) \\
& -v_{i}(t, x) \|^{2},
\end{aligned}
$$

$$
\begin{aligned}
& \mathbb{E} \max _{i \in \mathcal{N}} \sup _{t \geqslant-\tau} \| \int_{0}^{t} e^{-q_{i}(t-\theta) \Delta} \\
& \cdot \sum_{j=1}^{n} h_{i j}\left(\int_{\theta-\rho(\theta)}^{\theta}\left[f_{j}\left(u_{j}(s, x)\right)-f_{j}\left(v_{j}(s, x)\right)\right] d s\right) d \theta \|^{2} \\
& \leqslant n M^{2} \tau^{2} \frac{1}{\gamma^{2}} \max _{i \in \mathcal{N}}\left(\sum_{j=1}^{n}\left|h_{i j}\right|^{2} L_{j}^{2}\right) \mathbb{E} \max _{i \in \mathcal{N}} \sup _{t \geqslant-\tau} \| u_{i}(t, x) \\
& -v_{i}(t, x) \|^{2}, \\
& \mathbb{E} \max _{i \in \mathcal{N}} \sup _{t \geqslant-\tau} \| e^{-q_{i} t \Delta} \sum_{0<t_{k}<t} e^{q_{i} t_{k} \Delta} \\
& \cdot\left[g_{i}\left(u_{i}\left(t_{k}, x\right)\right)-g_{i}\left(v_{i}\left(t_{k}, x\right)\right)\right] \|^{2} \leqslant M^{4}\left(\max _{i \in \mathcal{N}} G_{i}^{2}\right) \\
& \cdot\left[2 \mathbb{E} \max _{i \in \mathcal{N}} \sup _{t \geqslant-\tau}\left\|u_{i}(t, x)-v_{i}(t, x)\right\|^{2}\right. \\
& \left.+2 \mathbb{E} \max _{i \in \mathcal{N}} \sup _{t \geqslant-\tau}\left(\frac{1}{\mu} e^{-\gamma t} \int_{0}^{t} e^{\gamma s}\left\|u_{i}(s, x)-v_{i}(s, x)\right\| d s\right)^{2}\right] \\
& \leqslant 2 M^{4}\left(1+\frac{1}{\gamma^{2} \mu^{2}}\right)\left(\max _{i \in \mathcal{N}} G_{i}^{2}\right) \mathbb{E} \max _{i \in \mathcal{N}} \sup _{t \geqslant-\tau} \| u_{i}(t, x) \\
& -v_{i}(t, x) \|^{2},
\end{aligned}
$$

where we assume that $t_{j-1}<t \leqslant t_{j}$.

In addition, it follows from Burkholder-Davis-Gundy inequality that

$$
\begin{aligned}
\mathbb{E} \max _{i \in \mathcal{N}} \sup _{t \geqslant-\tau} \| \int_{0}^{t} e^{-q_{i}(t-\theta) \Delta} \\
\cdot\left[\sigma_{i}\left(u_{i}(\theta, x)\right)-\sigma_{i}\left(v_{i}(\theta, x)\right)\right] d w_{i}(\theta) \|^{2} \leqslant \frac{2}{\gamma} \\
\cdot M^{2}\left(\max _{i \in \mathcal{N}} T_{i}^{2}\right) \mathbb{E} \max _{i \in \mathcal{N}} \sup _{t \geqslant-\tau}\left\|u_{i}(\theta, x)-v_{i}(\theta, x)\right\|^{2} .
\end{aligned}
$$

Now, combining (37)-(39) gives

$$
\operatorname{dist}(\Theta(u), \Theta(v)) \leqslant \sqrt{\kappa} \operatorname{dist}(u, v),
$$

$$
\forall u, v \in \Gamma_{1} \times \Gamma_{2} \times \cdots \times \Gamma_{n},
$$

where $\kappa$ is defined as (6), satisfying $0<\kappa<1$. This implies that $\Theta: \Gamma_{1} \times \Gamma_{2} \times \cdots \times \Gamma_{n} \rightarrow \Gamma_{1} \times \Gamma_{2} \times$ $\cdots \times \Gamma_{n}$ is a contraction mapping such that there exists the fixed point $u \triangleq\left(u_{1}(t, x), u_{2}(t, x), \ldots, u_{n}(t, x)\right)$ of $\Theta$ in $\Gamma_{1} \times \Gamma_{2} \times \cdots \times \Gamma_{n}$, which implies that $u$ is a solution of CNN (1), satisfying $\mathbb{E}\left(e^{\alpha t}\left\|\Theta_{i}\left(u_{i}(t, x)\right)\right\|^{2}\right) \rightarrow 0, t \rightarrow$ $+\infty$ so that $\mathbb{E m a x}_{i \in \mathcal{N}} \sup _{t \geqslant-\tau}\left(e^{\alpha t}\left\|\Theta_{i}\left(u_{i}(t, x)\right)\right\|^{2}\right) \rightarrow 0, t \rightarrow$ $+\infty$. Therefore, CNN (1) is stochastically exponentially mean square stable. 
TABLE 1: Comparison of the complexity of system models in the literature related to fixed point theory.

\begin{tabular}{|c|c|c|c|c|c|}
\hline & Theorem 5 & [16] & [15] & {$[23]$} & {$[24]$} \\
\hline Using fixed point theory & Yes & Yes & Yes & Yes & Yes \\
\hline Impulse model & Yes & No & No & Yes & Yes \\
\hline Distributed delays & Yes & No & No & No & No \\
\hline Reaction-diffusion model & Yes & No & No & No & No \\
\hline Itô stochastic model & Yes & No & No & No & No \\
\hline Equations type & Integrodifferential (partial) eq. & Differential eq. & Differential eq. & Differential eq. & Integrodifferential eq. \\
\hline Stability type & Stochastically exponential & Exponential & Exponential & Exponential & Exponential \\
\hline
\end{tabular}

\section{Numerical Example}

Consider the following impulsive stochastic reaction-diffusion CNN with distributed delay:

$$
\begin{aligned}
& d u_{i}(t, x)=-q_{i} \operatorname{div} \nabla u_{i}(t, x) d t-\left[a_{i} u_{i}(t, x)\right. \\
& \quad-\sum_{j=1}^{n} b_{i j} \sin \left(\frac{j}{10} u_{j}(t, x)\right) \\
& \quad-\sum_{j=1}^{n} c_{i j} \sin \frac{j}{10}\left(u_{j}(t-\tau(t), x)\right) \\
& \left.\quad-\sum_{j=1}^{n} h_{i j} \int_{t-\rho(t)}^{t} \sin \left(\frac{j}{10} u_{j}(s, x)\right) d s\right] d t \\
& +\sin \left(0.05 i u_{i}(t, x)\right) d w_{i}(t), \quad t \neq t_{k}, x \in \Upsilon, i \in \mathcal{N} \\
& u\left(t_{k}^{+}, x\right)=u\left(t_{k}^{-}, x\right)+0.1 j \sin \left(u\left(t_{k}, x\right)\right), \\
& u_{i}(t, x)=\zeta_{i}(t, x), \quad(s, x) \in[-\tau, 0] \times \Upsilon \\
& u(t, x)=0, \quad u \in[0,+\infty) \times \partial \Upsilon, \quad k=1,2, \ldots
\end{aligned}
$$

where we suppose $\Upsilon=(0, \pi), n=2, \tau=3, \mu=1.5, a_{i}=0.5 i$, $b_{i j}=0.01(i+j)=c_{i j}=h_{i j}$, and $q_{i}=-1$. Then, via computing the eigenfunctions of $-\Delta$, we can obtain that $\left\|e^{t \Delta}\right\| \leqslant e^{-\pi^{2} t}, t \geqslant$ 0 , so that we can take $\gamma=\pi^{2}, M=1$. In addition, differential mean value theorem yields

$$
\begin{aligned}
& \left|\sin \left(\frac{j}{10} u_{j}(t, x)\right)-\sin \left(\frac{j}{10} v_{j}(t, x)\right)\right| \\
& \quad \leqslant \frac{j}{10}\left|u_{j}(t, x)-v_{j}(t, x)\right|,
\end{aligned}
$$

and then we get $L_{j}=j / 10, j=1,2$. Similarly, we can compute that $G_{i}=0.1 i, T_{i}=0.05 i$, and $i=1,2$. Finally, we can compute (6) on a computer running Matlab software, obtaining $\kappa=0.8716 \in(0,1)$. Therefore, Theorem 5 tells us that CNN (41) is stochastically exponentially mean square stable.
Table 1 is presented to compare the complexity of neural networks investigated in various literatures via fixed point theorems and techniques.

Remark 6. Impulsive reaction-diffusion Itô-type stochastic model gives a lot of mathematical difficulties in deriving the stability criterion. Motivated by some methods and techniques of the above-mentioned literature ([3-31]), this is the first time for us to analyze such a complex model by way of fixed point theorem. Our model is closer to real engineering so that it is more complex than those of the previous literature, and we utilize Banach fixed point theorem, Hölder inequality, Burkholder-Davis-Gundy inequality, and the continuous semigroup of Laplace operators to overcome the difficulties. Besides, the distance defined in this paper satisfies the triangle inequality, which is another point different from those of previous related literatures.

\section{Conclusions}

Since our CNN model involves pulse and Laplacian operators, our model is different from the previous model ([15-22]), which also implies some difficulties in mathematical techniques. Motivated by the previous literature related to fixed point theory ([15-22, 25-31]), the authors employed Banach fixed point theorem, Hölder inequality, Burkholder-Davis-Gundy inequality, and the continuous semigroup of Laplace operators to derive the stochastically exponential stability criterion of impulsive stochastic reaction-diffusion cellular neural networks with distributed delay.

\section{Conflicts of Interest}

The authors declare that they have no conflicts of interest.

\section{Acknowledgments}

This work was supported by the National Basic Research Program of China (2010CB732501), Scientific Research Fund of Science Technology Department of Sichuan Province (2010JY0057, 2012JY010), Sichuan Educational Committee Science Foundation (08ZB002, 12ZB349, 14ZA0274), and the Initial Founding of Scientific Research for Chengdu Normal University Introduction of Talents. 


\section{References}

[1] L. O. Chua and L. Yang, "Cellular neural networks: theory," Institute of Electrical and Electronics Engineers. Transactions on Circuits and Systems, vol. 35, no. 10, pp. 1257-1272, 1988.

[2] L. O. Chua and L. Yang, "Cellular neural networks: applications," Institute of Electrical and Electronics Engineers. Transactions on Circuits and Systems, vol. 35, no. 10, pp. 1273-1290, 1988.

[3] X. Li and R. Rakkiyappan, "Impulse controller design for exponential synchronization of chaotic neural networks with mixed delays," Communications in Nonlinear Science and Numerical Simulation, vol. 18, no. 6, pp. 1515-1523, 2013.

[4] B. Wang, J. Cheng, and J. Zhan, "A sojourn probability approach to fuzzy-model-based reliable control for switched systems with mode-dependent time-varying delays," Nonlinear Analysis. Hybrid Systems, vol. 26, pp. 239-253, 2017.

[5] X. Li, R. Rakkiyappan, and P. Balasubramaniam, "Existence and global stability analysis of equilibrium of fuzzy cellular neural networks with time delay in the leakage term under impulsive perturbations," Journal of the Franklin Institute. Engineering and Applied Mathematics, vol. 348, no. 2, pp. 135-155, 2011.

[6] Q. Song, H. Yan, Z. Zhao, and Y. Liu, "Global exponential stability of impulsive complex-valued neural networks with both asynchronous time-varying and continuously distributed delays," Neural Networks, vol. 81, pp. 1-10, 2016.

[7] M. Kimura, R. Morita, S. Sugisaki, T. Matsuda, and Y. Nakashima, "Cellular neural network formed by simplified processing elements composed of thin-film transistors," Neurocomputing, vol. 248, pp. 112-119, 2017.

[8] J. Cheng, J. H. Park, Y. Liu, Z. Liu, and L. Tang, "Finite-time H $\infty$ fuzzy control of nonlinear Markovian jump delayed systems with partly uncertain transition descriptions," Fuzzy Sets and Systems, vol. 314, pp. 99-115, 2017.

[9] X. Li, C. Ding, and Q. Zhu, "Synchronization of stochastic perturbed chaotic neural networks with mixed delays," Journal of the Franklin Institute. Engineering and Applied Mathematics, vol. 347, no. 7, pp. 1266-1280, 2010.

[10] B. Wang, J. Cheng, A. Al-Barakati, and H. M. Fardoun, "A mismatched membership function approach to sampled-data stabilization for T-S fuzzy systems with time-varying delayed signals," Signal Processing, vol. 140, pp. 161-170, 2017.

[11] K. Shi, X. Liu, Y. Tang, H. Zhu, and S. Zhong, "Some novel approaches on state estimation of delayed neural networks," Information Sciences, vol. 372, pp. 313-331, 2016.

[12] Q. Song and J. Cao, "Dynamical behaviors of discrete-time fuzzy cellular neural networks with variable delays and impulses," Journal of the Franklin Institute. Engineering and Applied Mathematics, vol. 345, no. 1, pp. 39-59, 2008.

[13] R. Jia, "Finite-time stability of a class of fuzzy cellular neural networks with multi-proportional delays," Fuzzy Sets and Systems. An International Journal in Information Science and Engineering, vol. 319, pp. 70-80, 2017.

[14] Y. G. Kao, L. Shi, J. Xie, and H. R. Karimi, "Global exponential stability of delayed Markovian jump fuzzy cellular neural networks with generally incomplete transition probability," Neural Networks, vol. 63, pp. 18-30, 2015.

[15] B. Liu, "Global exponential stability for BAM neural networks with time-varying delays in the leakage terms," Nonlinear Analysis. Real World Applications. An International Multidisciplinary Journal, vol. 14, no. 1, pp. 559-566, 2013.
[16] L. Zhou, "Novel global exponential stability criteria for hybrid BAM neural networks with proportional delays," Neurocomputing, vol. 161, pp. 99-106, 2015.

[17] J. Luo, "Fixed points and stability of neutral stochastic delay differential equations," Journal of Mathematical Analysis and Applications, vol. 334, no. 1, pp. 431-440, 2007.

[18] G. Chen, O. van Gaans, and S. Verduyn Lunel, "Fixed points and pth moment exponential stability of stochastic delayed recurrent neural networks with impulses," Applied Mathematics Letters. An International Journal of Rapid Publication, vol. 27, pp. 36-42, 2014.

[19] C. Guo, D. O’Regan, F. Deng, and R. P. Agarwal, "Fixed points and exponential stability for a stochastic neutral cellular neural network," Applied Mathematics Letters. An International Journal of Rapid Publication, vol. 26, no. 8, pp. 849-853, 2013.

[20] X. Yang, Q. Zhu, and Z. Yao, "pth Moment Exponential Stability of Nonlinear Hybrid Stochastic Heat Equations," Mathematical Problems in Engineering, vol. 2014, Article ID 481246, 7 pages, 2014.

[21] R. Rao and Z. Pu, "LMI-based stability criterion of impulsive TS fuzzy dynamic equations via fixed point theory," Abstract and Applied Analysis, vol. 2013, Article ID 261353, 2013.

[22] G.-Q. Wang and S. S. Cheng, "Fixed point theorems arising from seeking steady states of neural networks," Applied Mathematical Modelling. Simulation and Computation for Engineering and Environmental Systems, vol. 33, no. 1, pp. 499-506, 2009.

[23] Y. Zhang and Q. Luo, "Global exponential stability of impulsive cellular neural networks with time-varying delays via fixed point theory," Advances in Difference Equations, vol. 2013, 23 pages, 2013.

[24] R. Rao, S. Zhong, and Z. Pu, "LMI-based robust exponential stability criterion of impulsive integro-differential equations with uncertain parameters via contraction mapping theory," Advances in Difference Equations, vol. 2017, 19 pages, 2017.

[25] J. Luo, "Exponentially stable stationary solutions for delay stochastic evolution equations," Progress in Probability, vol. 65, pp. 169-178, 2011.

[26] A. A. Kwiecinska, "Stabilization of partial differential equations by noise," Stochastic Processes and their Applications, vol. 79, no. 2, pp. 179-184, 1999.

[27] R. Rao and Z. Pu, "Stability analysis for impulsive stochastic fuzzy p-Laplace dynamic equations under Neumann or Dirichlet boundary condition," Boundary Value Problems, vol. 2013, 14 pages, 2013.

[28] Q. Zhu, X. Li, and X. Yang, "Exponential stability for stochastic reaction-diffusion BAM neural networks with time-varying and distributed delays," Applied Mathematics and Computation, vol. 217, no. 13, pp. 6078-6091, 2011.

[29] R. Rao, S. Zhong, and Z. Pu, "On the role of diffusion factors in stability analysis for p-Laplace dynamical equations involved to BAM Cohen-Grossberg neural network," Neurocomputing, vol. 223, pp. 54-62, 2017.

[30] B. Xie, "The moment and almost surely exponential stability of stochastic heat equations," Proceedings of the American Mathematical Society, vol. 136, no. 10, pp. 3627-3634, 2008.

[31] X. Li and F. Deng, "Razumikhin method for impulsive functional differential equations of neutral type," Chaos, Solitons \& Fractals, vol. 101, pp. 41-49, 2017. 


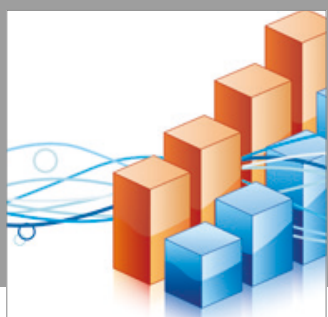

Advances in

Operations Research

vatersals

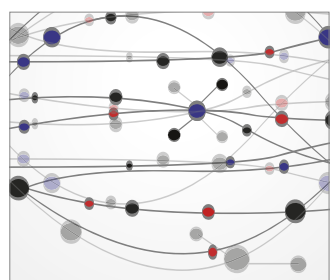

\section{The Scientific} World Journal
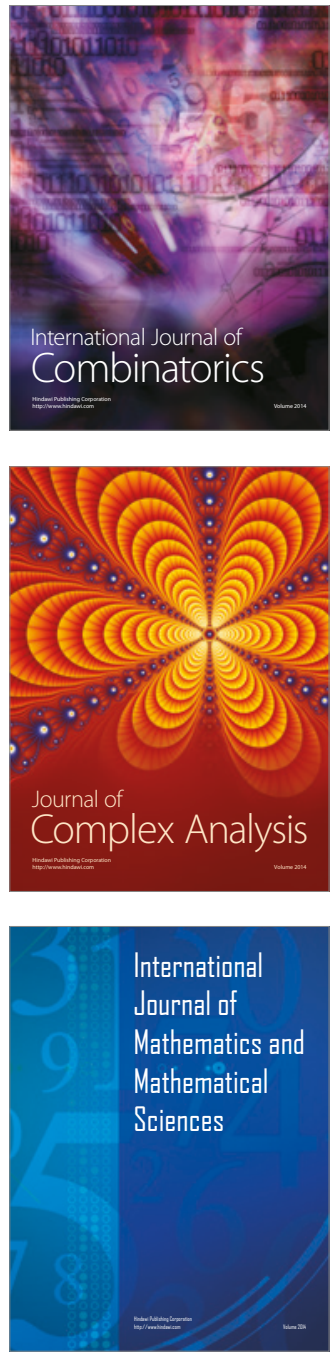
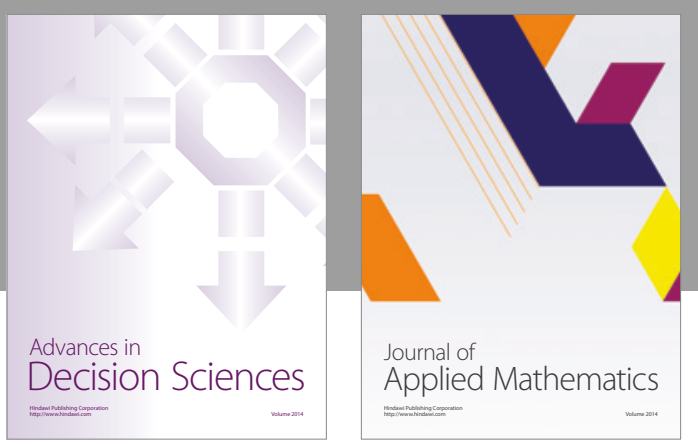

Algebra

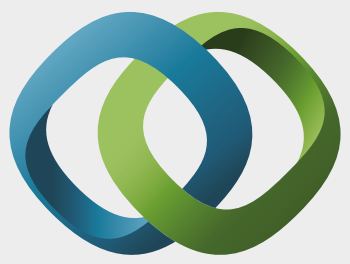

\section{Hindawi}

Submit your manuscripts at

https://www.hindawi.com
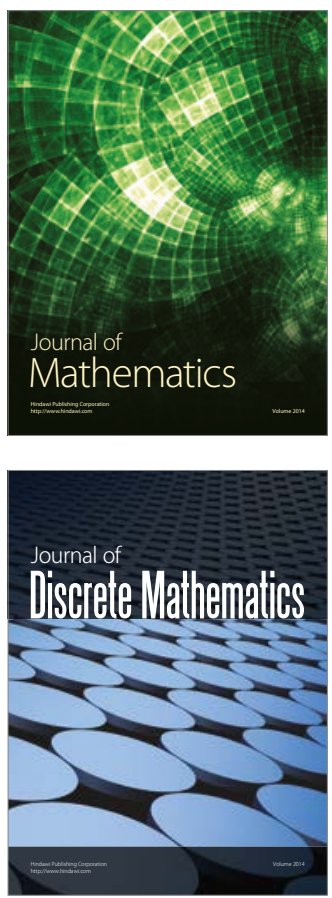

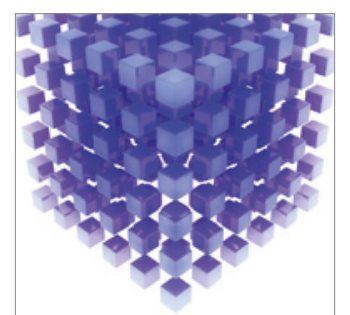

Mathematical Problems in Engineering
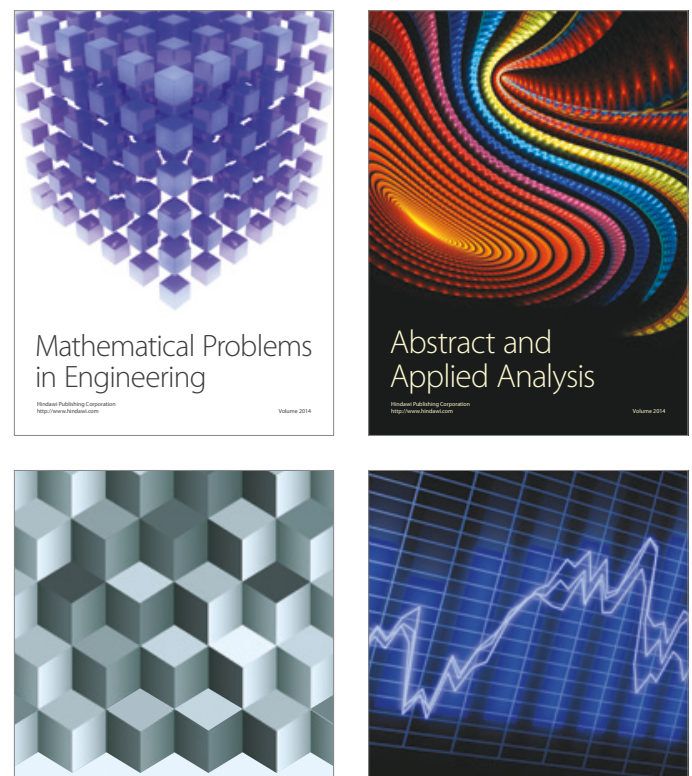

Journal of

Function Spaces

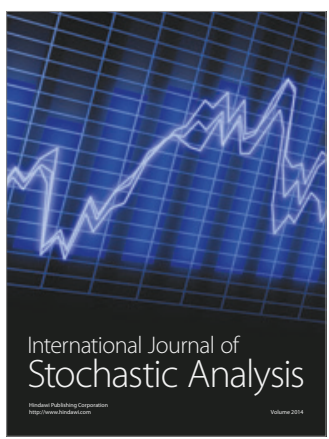

Probability and Statistics
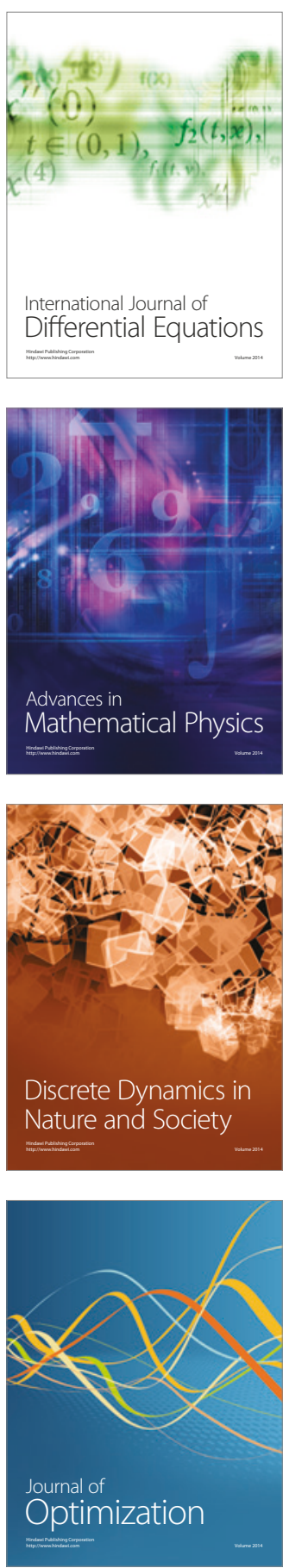